\title{
Existence of nonlinear Lane-Emden equation offractional order
}

\author{
Rabha W. Ibrahim
}




\title{
EXISTENCE OF NONLINEAR LANE-EMDEN EQUATION OF FRACTIONAL ORDER
}

\author{
RABHA W. IBRAHIM
}

Received 19 December, 2011

\begin{abstract}
We study a Dirichlet boundary value problem for the Lane-Emden equation involving two fractional orders. Lane-Emden equation has been widely used to describe a variety of phenomena in physics and astrophysics, including aspects of stellar structure, the thermal history of a spherical cloud of gas, isothermal gas spheres, and thermionic currents. However, ordinary Lane-Emden equation does not provide a correct description of the dynamics of systems in complex media. In order to overcome this problem and describe dynamical processes in a fractal medium, numerous generalizations of Lane-Emden equation have been proposed. One such generalization replaces the ordinary derivative by a fractional derivative in the Lane-Emden equation. This gives rise to the fractional Lane-Emden equation with a single index. Recently, a new type of Lane-Emden equation with two different fractional orders has been introduced which provides a more flexible model for fractal processes as compared with the usual one characterized by a single index. The contraction mapping principle and Krasnoselskii's fixed point theorem are applied to prove the existence of solutions of the problem in a Banach space.
\end{abstract}

2000 Mathematics Subject Classification: 34G20; 26A33; 34A12

Keywords: fractional calculus, fractional differential equation, Lane-Emden equation, RiemannLiouville fractional operators, Volterra integral equation

\section{INTRODUCTION}

The theory of singular boundary value problems has become an important area of investigation in the past three decades (see $[2-5,10])$. One of the equations arising in this topic is the Lane-Emden equation. Lane-Emden type equations, first published by Jonathan Homer Lane in 1870 [18], and further explored in detail by Emden [11], represents such phenomena and have significant applications. It is a second- order ordinary differential equation with an arbitrary index, known as the polytropic index, that occurs in one of its terms. The Lane-Emden equation describes a variety of phenomena in physics and astrophysics, including aspects of stellar structure, the thermal history of a spherical cloud of gas, isothermal gas spheres and thermionic currents [8]. 
The solution of the Lane-Emden problem, as well as other various linear and nonlinear singular initial value problems in quantum mechanics and astrophysics, is numerically challenging because of the singularity behavior at the origin. The approximate solutions of the Lane-Emden equation were given by homotopy perturbation method [9], variational iteration method [26], and Sinc-Collocation method [23], an implicit series solution [21]. Recently, Parand et. al [22] proposed an approximation algorithm for the solution of the nonlinear Lane-Emden type equation using Hermite functions collocation method. Moreover, Adibi and Rismani [1] introduced a modified Legendre-spectral method. Finally, Bhrawy and Alofi ][6] imposed a Jacobi-Gauss collocation method for solving nonlinear Lane-Emden type equations.

Lane-Emden equations have the following form

$$
u^{\prime \prime}(t)+\frac{a}{t} u^{\prime}(t)+f(t, u)=g(t), \quad 0<t \leq 1, a \geq 0
$$

with the initial condition

$$
u(0)=A, \quad u^{\prime}(0)=B,
$$

where $A, B$ are constants, $f(t, u)$ is a continuous real valued function and $g(t) \in$ $C[0,1]$.

\section{Fractional Calculus}

Fractional calculus and its applications (that is the theory of derivatives and integrals of any arbitrary real or complex order) has importance in diverse areas of mathematical physics and engineering sciences. It generalized the ideas of integer order differentiation and n-fold integration. Fractional derivatives introduce an excellent instrument for the description of general properties of various materials and processes. This is the main advantage of fractional derivatives in comparison with classical integer-order models, in which such effects are in fact neglected. The advantages of fractional derivatives become apparent in modeling mechanical and electrical properties of real materials, as well as in the description of properties of gases, liquids and rocks, and in many other fields (see [19,27]).

The class of fractional differential equations of various types plays important role and is an useful tool not only in mathematics but also in physics, control systems, dynamical systems and engineering in order to create the mathematical models of many physical phenomena. Naturally, such equations are desired to be solved. Many studies on fractional calculus and fractional differential equations, involving different operators such as Riemann-Liouville operators, Erdélyi-Kober operators, Weyl-Riesz operators, Caputo operators and Grünwald-Letnikov operators, have appeared during the past three decades. The existence of positive solution and multi-positive solutions for nonlinear fractional differential equation are established and studied [7, 12, 15, 16, 20, 24]. Moreover, by using the concepts of subordination and superordination of analytic functions, the existence of analytic solutions for fractional differential equations in complex domain are suggested and posed in [13,14]. 
One of the most frequently used tools in the theory of fractional calculus is the the Riemann-Liouville operator (see [24]). The Riemann-Liouville fractional derivative are well posed for the physical interpretation of the initial conditions required for the initial value problems involving fractional differential equations. Moreover, this operator possesses advantages of fast convergence, higher stability and higher accuracy to derive different types of numerical algorithms (see [17]).

Definition 1. The fractional (arbitrary) order integral of the function $f$ of order $\alpha>0$ is defined by

$$
I_{a}^{\alpha} f(t)=\int_{a}^{t} \frac{(t-\tau)^{\alpha-1}}{\Gamma(\alpha)} f(\tau) d \tau
$$

When $a=0$, we write $I_{a}^{\alpha} f(t)=f(t) * \phi_{\alpha}(t)$, where $(*)$ denotes the convolution product (see [24]), $\phi_{\alpha}(t)=\frac{t^{\alpha-1}}{\Gamma(\alpha)}, t>0$ and $\phi_{\alpha}(t)=0, t \leq 0$ and $\phi_{\alpha} \rightarrow \delta(t)$ as $\alpha \rightarrow 0$ where $\delta(t)$ is the delta function.

Definition 2. The fractional (arbitrary) order derivative of the function $f$ of order $0 \leq \alpha<1$ is defined by

$$
D_{a}^{\alpha} f(t)=\frac{d}{d t} \int_{a}^{t} \frac{(t-\tau)^{-\alpha}}{\Gamma(1-\alpha)} f(\tau) d \tau=\frac{d}{d t} I_{a}^{1-\alpha} f(t) .
$$

Remark 1. From Definition 1 and Definition 2, we have

and

$$
D^{\alpha} t^{\mu}=\frac{\Gamma(\mu+1)}{\Gamma(\mu-\alpha+1)} t^{\mu-\alpha}, \mu>-1 ; 0<\alpha<1
$$

$$
I^{\alpha} t^{\mu}=\frac{\Gamma(\mu+1)}{\Gamma(\mu+\alpha+1)} t^{\mu+\alpha}, \mu>-1 ; \alpha>0 .
$$

In this note, we consider the fractional Lane-Emden equations of the following form

$$
\begin{gathered}
D^{\beta}\left(D^{\alpha}+\frac{a}{t}\right) u(t)+f(t, u)=g(t), \\
(0<t \leq 1, a \geq 0,0<\alpha, \beta \leq 1)
\end{gathered}
$$

with boundary conditions

$$
u(0)=u(\mu)=u(1)=0, \quad 0<\mu<1,
$$

where $f(t, u)$ is a continuous real valued function and $g(t) \in C[0,1]$.

Now, we state a known result due to Krasnoselskii [25] which is needed to prove the existence of solution.

Theorem 1. Let $M$ be a closed convex and nonempty subset of a Banach space $X$. Let $A, B$ be the operators such that (i) $A x+B y \in M$ whenever $x, y \in M$; (ii) $A$ is compact and continuous; (iii) $B$ is a contraction mapping. Then there exists $z \in M$ such that $z=A z+B z$. 


\section{Existence OF SOLUTIONS}

In this section, we establish the solution of Eq. 2.1. Assume that $\mathscr{B}$ is a Banach space $X$ of all continues bounded functions endowed with the supremum norm. We introduce the following linear problem for the Lane-Emden equation involving two fractional orders:

$$
\begin{gathered}
D^{\beta}\left(D^{\alpha}+\frac{a}{t}\right) u(t)=g(t), \\
(0<t \leq 1, a \geq 0,0<\alpha, \beta \leq 1)
\end{gathered}
$$

with the boundary conditions

$$
u(0)=u(\mu)=u(1)=0, \quad 0<\mu<1 .
$$

Lemma 1. A unique solution of the linear three-point boundary value problem for the Lane-Emden equation 3.1 is given by

$$
\begin{aligned}
u(t) & =\int_{0}^{t} \frac{(t-\tau)^{\alpha-1}}{\Gamma(\alpha)}\left(\int_{0}^{\tau} \frac{(\tau-s)^{\beta-1}}{\Gamma(\beta)} g(s) d s-\frac{a}{\tau} u(\tau)\right) d \tau \\
& +t^{\alpha} \frac{(t-\mu)}{1-\mu}\left[\int_{0}^{1} \frac{(1-\tau)^{\alpha-1}}{\Gamma(\alpha)}\left(\int_{0}^{\tau} \frac{(\tau-s)^{\beta-1}}{\Gamma(\beta)} g(s) d s-\frac{a}{\tau} u(\tau)\right) d \tau\right] \\
& -\frac{t^{\alpha}(1-t)}{\mu^{\alpha}(1-\mu)}\left[\int_{0}^{\mu} \frac{(\mu-\tau)^{\alpha-1}}{\Gamma(\alpha)}\left(\int_{0}^{\tau} \frac{(\tau-s)^{\beta-1}}{\Gamma(\beta)} g(s) d s-\frac{a}{\tau} u(\tau)\right) d \tau\right],
\end{aligned}
$$

where $g \in C[0,1]$.

Proof. The general solution of

$$
D^{\beta}\left(D^{\alpha}+\frac{a}{t}\right) u(t)=g(t)
$$

can be written as

$$
\begin{gathered}
u(t)=\int_{0}^{t} \frac{(t-\tau)^{\alpha-1}}{\Gamma(\alpha)}\left(\int_{0}^{\tau} \frac{(\tau-s)^{\beta-1}}{\Gamma(\beta)} g(s) d s-\frac{a}{\tau} u(\tau)\right) d \tau \\
-\frac{c_{1}}{\Gamma(\alpha+2)} t^{\alpha+1}-\frac{c_{2}}{\Gamma(\alpha+1)} t^{\alpha}-c_{3} .
\end{gathered}
$$


Using the boundary conditions for 3.1 we obtain

$$
\begin{aligned}
c_{3} & =0, \\
c_{2} & =\frac{\mu(\alpha+1)}{(1-\mu)}\left[\int_{0}^{1}(1-\tau)^{\alpha-1}\left(\int_{0}^{\tau} \frac{(\tau-s)^{\beta-1}}{\Gamma(\beta)} g(s) d s-\frac{a}{\tau} u(\tau)\right) d \tau\right] \\
& -\frac{1}{\mu^{\alpha+1}}\left[\int_{0}^{\mu}(\mu-\tau)^{\alpha-1}\left(\int_{0}^{\tau} \frac{(\tau-s)^{\beta-1}}{\Gamma(\beta)} g(s) d s-\frac{a}{\tau} u(\tau)\right) d \tau\right] \\
c_{1} & =\frac{(\alpha+2)(\alpha+1)}{1-\mu}\left[\int_{0}^{1}(1-\tau)^{\alpha-1}\left(\int_{0}^{\tau} \frac{(\tau-s)^{\beta-1}}{\Gamma(\beta)} g(s) d s-\frac{a}{\tau} u(\tau)\right) d \tau\right. \\
& \left.-\frac{1}{\mu^{\alpha}} \int_{0}^{\mu}(\mu-\tau)^{\alpha-1}\left(\int_{0}^{\tau} \frac{(\tau-s)^{\beta-1}}{\Gamma(\beta)} g(s) d s-\frac{a}{\tau} u(\tau)\right) d \tau\right]
\end{aligned}
$$

Substituting the last assertions in 3.3, we obtain the solution given by 3.2. This completes the proof.

Theorem 2. Let $f:[0,1] \times X \longrightarrow X$ be a jointly continuous function satisfying the condition

$$
|f(t, u)-f(t, v)| \leq L|u-v|, \quad \forall t \in[0,1], u, v \in X .
$$

Moreover, assume that $\sup _{t \in[0,1]}|g(t)|=\gamma$. Then the boundary value problem 2.1 has a unique solution provided by

$$
\ell:=\frac{L}{\Gamma(\alpha+\beta+1)}\left(2+\mu^{\alpha+\beta}\right)+a \frac{\Gamma(\alpha)}{\Gamma(2 \alpha)}\left(2+\mu^{2 \alpha-1}\right)<1, \quad a \geq 0 .
$$

Proof. Define $P: X \rightarrow X$ by

$$
\begin{gathered}
(P u)(t)=\int_{0}^{t} \frac{(t-\tau)^{\alpha-1}}{\Gamma(\alpha)}\left(\int_{0}^{\tau} \frac{(\tau-s)^{\beta-1}}{\Gamma(\beta)}(g(s)-f(s, u(s))) d s-\frac{a}{\tau} u(\tau)\right) d \tau \\
+t^{\alpha} \frac{(t-\mu)}{1-\mu} \\
{\left[\int_{0}^{1} \frac{(1-\tau)^{\alpha-1}}{\Gamma(\alpha)}\left(\int_{0}^{\tau} \frac{(\tau-s)^{\beta-1}}{\Gamma(\beta)}(g(s)-f(s, u(s))) d s-\frac{a}{\tau} u(\tau)\right) d \tau\right]} \\
-\frac{t^{\alpha}(1-t)}{\mu^{\alpha}(1-\mu)} \\
{\left[\int_{0}^{\mu} \frac{(\mu-\tau)^{\alpha-1}}{\Gamma(\alpha)}\left(\int_{0}^{\tau} \frac{(\tau-s)^{\beta-1}}{\Gamma(\beta)}(g(s)-f(s, u(s))) d s-\frac{a}{\tau} u(\tau)\right) d \tau\right]}
\end{gathered}
$$

Setting $\sup _{t \in[0,1]}|f(t, u)|=M$ and we chose

$$
r \geq \frac{1}{1-\epsilon}\left(\frac{\gamma+M}{\Gamma(\alpha+\beta+1)}\left(2+\mu^{\alpha+\beta}\right)\right)
$$


where $\epsilon$ such that $\ell \leq \epsilon<1$. Now we show that $P B_{r} \subset B_{r}$, where $B_{r}:=\{u \in X$ : $\|u\| \leq r\}$. For $u \in X$, we have

$$
\begin{aligned}
& \|(P u)(t)\| \\
& =\sup _{t \in[0,1]} \mid \int_{0}^{t} \frac{(t-\tau)^{\alpha-1}}{\Gamma(\alpha)}\left(\int_{0}^{\tau} \frac{(\tau-s)^{\beta-1}}{\Gamma(\beta)}(g(s)-f(s, u(s))) d s-\frac{a}{\tau} u(\tau)\right) d \tau \\
& +t^{\alpha} \frac{(t-\mu)}{1-\mu}\left[\int_{0}^{1} \frac{(1-\tau)^{\alpha-1}}{\Gamma(\alpha)}\left(\int_{0}^{\tau} \frac{(\tau-s)^{\beta-1}}{\Gamma(\beta)}(g(s)-f(s, u(s))) d s-\frac{a}{\tau} u(\tau)\right) d \tau\right] \\
& -\frac{t^{\alpha}(1-t)}{\mu^{\alpha}(1-\mu)} \\
& {\left[\int_{0}^{\mu} \frac{(\mu-\tau)^{\alpha-1}}{\Gamma(\alpha)}\left(\int_{0}^{\tau} \frac{(\tau-s)^{\beta-1}}{\Gamma(\beta)}(g(s)-f(s, u(s))) d s-\frac{a}{\tau} u(\tau)\right) d \tau\right]} \\
& \leq \sup _{t \in[0,1]} \int_{0}^{t} \frac{(t-\tau)^{\alpha-1}}{\Gamma(\alpha)} \\
& \left(\int_{0}^{\tau} \frac{(\tau-s)^{\beta-1}}{\Gamma(\beta)}|g(s)-f(s, u(s))-f(s, 0)+f(s, 0)| d s+\left|\frac{a}{\tau} u(\tau)\right|\right) d \tau \\
& +\sup _{t \in[0,1]} t^{\alpha}\left|\frac{(t-\mu)}{1-\mu}\right|\left[\int_{0}^{1} \frac{(1-\tau)^{\alpha-1}}{\Gamma(\alpha)}\right. \\
& \left.\left(\int_{0}^{\tau} \frac{(\tau-s)^{\beta-1}}{\Gamma(\beta)}|g(s)-f(s, u(s))-f(s, 0)+f(s, 0)| d s+\left|\frac{a}{\tau} u(\tau)\right|\right) d \tau\right] \\
& +\sup _{t \in[0,1]} \frac{t^{\alpha}(1-t)}{\mu^{\alpha}(1-\mu)}\left[\int_{0}^{\mu} \frac{(\mu-\tau)^{\alpha-1}}{\Gamma(\alpha)}\right. \\
& \left.\left(\int_{0}^{\tau} \frac{(\tau-s)^{\beta-1}}{\Gamma(\beta)}|g(s)-f(s, u(s))-f(s, 0)+f(s, 0)| d s+\frac{a}{\tau}|u(\tau)|\right) d \tau\right] \\
& \leq \int_{0}^{1} \frac{(1-\tau)^{\alpha-1}}{\Gamma(\alpha)}\left(\int_{0}^{\tau} \frac{(\tau-s)^{\beta-1}}{\Gamma(\beta)} d s\right) d \tau[\gamma+M+L\|u\|] \\
& +\int_{0}^{1} \frac{(1-\tau)^{\alpha-1}}{\Gamma(\alpha)} \frac{1}{\tau} d \tau a\|u\| \\
& +\sup _{t \in[0,1]}\left(t^{\alpha}\left|\frac{(t-\mu)}{1-\mu}\right|\right) \int_{0}^{1} \frac{(1-\tau)^{\alpha-1}}{\Gamma(\alpha)}\left(\int_{0}^{\tau} \frac{(\tau-s)^{\beta-1}}{\Gamma(\beta)} d s\right) d \tau[\gamma+M+L\|u\|] \\
& +\sup _{t \in[0,1]}\left(t^{\alpha}\left|\frac{(t-\mu)}{1-\mu}\right|\right) \int_{0}^{1} \frac{(1-\tau)^{\alpha-1}}{\Gamma(\alpha)} \frac{1}{\tau} d \tau a\|u\| \\
& +\sup _{t \in[0,1]}\left(\frac{t^{\alpha}(1-t)}{\mu^{\alpha}(1-\mu)}\right)\left[\int_{0}^{\mu} \frac{(\mu-\tau)^{\alpha-1}}{\Gamma(\alpha)}\left(\int_{0}^{\tau} \frac{(\tau-s)^{\beta-1}}{\Gamma(\beta)} d s\right) d \tau[\gamma+M+L\|u\|]\right.
\end{aligned}
$$




$$
\begin{gathered}
+\sup _{t \in[0,1]}\left(\frac{t^{\alpha}(1-t)}{\mu^{\alpha}(1-\mu)}\right)\left[\int_{0}^{\mu} \frac{(\mu-\tau)^{\alpha-1}}{\Gamma(\alpha)} \frac{a}{\tau}\|u\| d \tau\right] \\
\leq(\gamma+M+L r)\left[2 \int_{0}^{1} \frac{(1-\tau)^{\alpha-1}}{\Gamma(\alpha)}\left(\int_{0}^{\tau} \frac{(\tau-s)^{\beta-1}}{\Gamma(\beta)} d s\right) d \tau\right. \\
\left.+\int_{0}^{\mu} \frac{(\mu-\tau)^{\alpha-1}}{\Gamma(\alpha)}\left(\int_{0}^{\tau} \frac{(\tau-s)^{\beta-1}}{\Gamma(\beta)} d s\right) d \tau\right] \\
+\operatorname{ar}\left[2 \int_{0}^{1} \frac{(1-\tau)^{\alpha-1}}{\Gamma(\alpha)} \tau^{\alpha-1} d \tau+\int_{0}^{\mu} \frac{(\mu-\tau)^{\alpha-1}}{\Gamma(\alpha)} \tau^{\alpha-1} d \tau\right] \\
=(\gamma+M+\operatorname{Lr})\left[\frac{2}{\Gamma(\alpha) \Gamma(\beta+1)} \int_{0}^{1}(1-\tau)^{\alpha-1} \tau^{\beta} d \tau+\int_{0}^{\mu} \frac{(\mu-\tau)^{\alpha-1}}{\Gamma(\alpha) \Gamma(\beta+1)} \tau^{\beta} d \tau\right] \\
+\operatorname{ar}\left[2 \int_{0}^{1} \frac{(1-\tau)^{\alpha-1}}{\Gamma(\alpha)} \tau^{\alpha-1} d \tau+\int_{0}^{\mu} \frac{(\mu-\tau)^{\alpha-1}}{\Gamma(\alpha)} \tau^{\alpha-1} d \tau\right] .
\end{gathered}
$$

Now by using 3.5, the relations for the Beta function

$$
\begin{gathered}
B(z, w)=\int_{0}^{1}(1-\tau)^{z-1} \tau^{w-1} d \tau=\frac{\Gamma(z) \Gamma(w)}{\Gamma(z+w)}, \\
B(z, w+1)=\int_{0}^{1}(1-\tau)^{z-1} \tau^{w} d \tau=\frac{\Gamma(z) \Gamma(w+1)}{\Gamma(z+w+1)}
\end{gathered}
$$

and

we find that

$$
\int_{0}^{\mu}(\mu-\tau)^{z-1} \tau^{w} d \tau=\frac{\Gamma(z) \Gamma(w+1)}{\Gamma(z+w+1)} \mu^{z+w}
$$

$$
\begin{gathered}
\|(P u)(t)\| \\
\leq(\gamma+M+L r)\left[\frac{2}{\Gamma(\alpha) \Gamma(\beta+1)} \int_{0}^{1}(1-\tau)^{\alpha-1} \tau^{\beta} d \tau+\int_{0}^{\mu} \frac{(\mu-\tau)^{\alpha-1}}{\Gamma(\alpha) \Gamma(\beta+1)} \tau^{\beta} d \tau\right] \\
+\operatorname{ar}\left[2 \int_{0}^{1} \frac{(1-\tau)^{\alpha-1}}{\Gamma(\alpha)} \tau^{\alpha-1} d \tau+\int_{0}^{\mu} \frac{(\mu-\tau)^{\alpha-1}}{\Gamma(\alpha) \Gamma(\beta+1)} \tau^{\beta} d \tau\right] \\
=\frac{\gamma+M+L r}{\Gamma(\alpha+\beta+1)}\left(2+\mu^{\alpha+\beta}\right)+\operatorname{ar} \frac{\Gamma(\alpha)}{\Gamma(2 \alpha)}\left(2+\mu^{2 \alpha-1}\right) \\
\leq(\ell+1-\epsilon) r \leq r .
\end{gathered}
$$

We proceed to prove that $P$ is a contraction mapping. For $u, v \in X$ and for all $t \in[0,1]$ we pose

$$
\begin{gathered}
\|(P u)(t)-(P v)(t)\| \\
=\sup _{t \in[0,1]}|(P u)(t)-(P v)(t)|
\end{gathered}
$$




$$
\begin{aligned}
& \leq \sup _{t \in[0,1]} \int_{0}^{t} \frac{(t-\tau)^{\alpha-1}}{\Gamma(\alpha)} \\
& \left(\int_{0}^{\tau} \frac{(\tau-s)^{\beta-1}}{\Gamma(\beta)}|f(s, u(s))-f(s, v(s))| d s+\frac{a}{\tau}|u(\tau)-v(\tau)|\right) d \tau \\
& +\sup _{t \in[0,1]} t^{\alpha}\left[\int_{0}^{1} \frac{(1-\tau)^{\alpha-1}}{\Gamma(\alpha)}\right. \\
& \left.\left(\int_{0}^{\tau} \frac{(\tau-s)^{\beta-1}}{\Gamma(\beta)}|f(s, u(s))-f(s, v(s))| d s+\frac{a}{\tau}|u(\tau)-v(\tau)|\right) d \tau\right] \\
& +\sup _{t \in[0,1]} \frac{t^{\alpha}(1-t)}{\mu^{\alpha}(1-\mu)}\left[\int_{0}^{\mu} \frac{(\mu-\tau)^{\alpha-1}}{\Gamma(\alpha)}\right. \\
& \left.\left(\int_{0}^{\tau} \frac{(\tau-s)^{\beta-1}}{\Gamma(\beta)}|f(s, u(s))-f(s, v(s))| d s+\frac{a}{\tau}|u(\tau)-v(\tau)|\right) d \tau\right] \\
& \leq \sup _{t \in[0,1]} \int_{0}^{t} \frac{(t-\tau)^{\alpha-1}}{\Gamma(\alpha)}\left(\int_{0}^{\tau} \frac{(\tau-s)^{\beta-1}}{\Gamma(\beta)} d s\right) d \tau[L\|u-v\|] \\
& +\sup _{t \in[0,1]} \int_{0}^{t} \frac{(t-\tau)^{\alpha-1}}{\Gamma(\alpha)} \frac{1}{\tau} d \tau a\|u-v\| \\
& +\sup _{t \in[0,1]} t^{\alpha} \int_{0}^{1} \frac{(1-\tau)^{\alpha-1}}{\Gamma(\alpha)}\left(\int_{0}^{\tau} \frac{(\tau-s)^{\beta-1}}{\Gamma(\beta)} d s\right) d \tau[L\|u-v\|] \\
& +\sup _{t \in[0,1]} t^{\alpha} \int_{0}^{1} \frac{(1-\tau)^{\alpha-1}}{\Gamma(\alpha)} \frac{1}{\tau} d \tau a\|u-v\| \\
& +\sup _{t \in[0,1]} \frac{t^{\alpha}(1-t)}{\mu^{\alpha}(1-\mu)}\left[\int_{0}^{\mu} \frac{(\mu-\tau)^{\alpha-1}}{\Gamma(\alpha)}\right. \\
& \left.\left(\int_{0}^{\tau} \frac{(\tau-s)^{\beta-1}}{\Gamma(\beta)}[L\|u-v\|] d s+\frac{a}{\tau}\|u-v\|\right) d \tau\right] \\
& \leq \ell\|u-v\|,
\end{aligned}
$$

then $P$ is a contraction. Thus, the conclusion of the theorem follows by the contraction mapping principle. This completes the proof.

Theorem 3. Let $f:[0,1] \times X \longrightarrow X$ be a jointly continuous function and maps bounded subsets of $[0,1] \times X$ into relatively compact subsets of $X$. Furthermore, assume that

$$
\begin{gathered}
|f(t, u)-f(t, v)| \leq L|u-v|, \quad \forall t \in[0,1], u, v \in X, \\
|f(t, u)| \leq \sigma(t), \quad \sigma \in\left(L^{1}[0,1], R^{+}\right)
\end{gathered}
$$


and $\sup _{t \in[0,1]}|g(t)|=\gamma$. If

$$
\left(\frac{L\left(1+\mu^{\alpha+\beta}\right)}{\Gamma(\alpha+\beta+1)}+\frac{a \Gamma(\alpha)\left(1+\mu^{2 \alpha-1}\right)}{\Gamma(2 \alpha)}\right)<1
$$

then the boundary value problem 2.1 has at least one solution on $[0,1]$.

Proof. Define two operators $A$ and $B$ on $B_{r}$ where

$$
r \geq \frac{\frac{\gamma+\|\sigma\|_{L} 1}{\Gamma(\alpha+\beta+1)}\left(2+\mu^{\alpha+\beta}\right)}{1-a \frac{2 \Gamma(\alpha)}{\Gamma(2 \alpha)}\left(2+\mu^{2 \alpha-1}\right)}
$$

as follows:

$$
(A u)(t):=\int_{0}^{t} \frac{(t-\tau)^{\alpha-1}}{\Gamma(\alpha)}\left(\int_{0}^{\tau} \frac{(\tau-s)^{\beta-1}}{\Gamma(\beta)}(g(s)-f(s, u(s))) d s-\frac{a}{\tau} u(\tau)\right) d \tau
$$

and

$$
\begin{gathered}
(B u)(t):= \\
t^{\alpha} \frac{(t-\mu)}{1-\mu}\left[\int_{0}^{1} \frac{(1-\tau)^{\alpha-1}}{\Gamma(\alpha)}\left(\int_{0}^{\tau} \frac{(\tau-s)^{\beta-1}}{\Gamma(\beta)}(g(s)-f(s, u(s))) d s-\frac{a}{\tau} u(\tau)\right) d \tau\right] \\
-\frac{t^{\alpha}(1-t)}{\mu^{\alpha}(1-\mu)}\left[\int_{0}^{\mu} \frac{(\mu-\tau)^{\alpha-1}}{\Gamma(\alpha)}\left(\int_{0}^{\tau} \frac{(\tau-s)^{\beta-1}}{\Gamma(\beta)}(g(s)-f(s, u(s))) d s-\frac{a}{\tau} u(\tau)\right) d \tau\right] .
\end{gathered}
$$

For $u, v \in B_{r}$ we obtain

$$
\begin{gathered}
\|A u+B v\| \\
\leq\left[\gamma+\|\sigma\|_{L^{1}}\right]\left[\frac{2}{\Gamma(\alpha+\beta+1)}+\frac{1}{\Gamma(\alpha+\beta+1)} \mu^{\alpha+\beta}\right] \\
+\operatorname{ar}\left[\frac{2 \Gamma(\alpha)}{\Gamma(2 \alpha)}+\frac{1}{\Gamma(\alpha+\beta+1)} \mu^{\alpha+\beta}\right] \\
\leq r .
\end{gathered}
$$

Thus, $A, B \in B_{r}$. Furthermore, for $u, v \in B_{r}$,

$$
\begin{gathered}
\|(B u)(t)-(B v)(t)\| \\
\leq \sup _{t \in[0,1]} t^{\alpha} \frac{(t-\mu)}{1-\mu}\left[\int_{0}^{1} \frac{(1-\tau)^{\alpha-1}}{\Gamma(\alpha)}\left(\int_{0}^{\tau} \frac{(\tau-s)^{\beta-1}}{\Gamma(\beta)} \mid f(s, u(s))\right)-f(s, v(s)) \mid d s\right. \\
\left.\left.+\frac{a}{\tau}|u(\tau)-v(\tau)|\right) d \tau\right] \\
+\sup _{t \in[0,1]} \frac{t^{\alpha}(1-t)}{\mu^{\alpha}(1-\mu)}\left[\int_{0}^{\mu} \frac{(\mu-\tau)^{\alpha-1}}{\Gamma(\alpha)}\left(\int_{0}^{\tau} \frac{(\tau-s)^{\beta-1}}{\Gamma(\beta)} \mid f(s, u(s))\right)-f(s, v(s)) \mid d s\right. \\
\left.\left.+\frac{a}{\tau}|u(\tau)-v(\tau)|\right) d \tau\right]
\end{gathered}
$$




$$
\begin{gathered}
\leq \sup _{t \in[0,1]} t^{\alpha} \int_{0}^{1} \frac{(1-\tau)^{\alpha-1}}{\Gamma(\alpha)}\left(\int_{0}^{\tau} \frac{(\tau-s)^{\beta-1}}{\Gamma(\beta)} d s\right) d \tau[L\|u-v\|] \\
+t^{\alpha} \int_{0}^{1} \frac{(1-\tau)^{\alpha-1}}{\Gamma(\alpha)} \frac{1}{\tau} d \tau a\|u-v\| \\
\quad+\sup _{t \in[0,1]} \frac{t^{\alpha}(1-t)}{\mu^{\alpha}(1-\mu)}\left[\int_{0}^{\mu} \frac{(\mu-\tau)^{\alpha-1}}{\Gamma(\alpha)}\right. \\
\left.\left(\int_{0}^{\tau} \frac{(\tau-s)^{\beta-1}}{\Gamma(\beta)}[L\|u-v\|] d s+\frac{a}{\tau}\|u-v\|\right) d \tau\right] \\
\leq[L\|u-v\|] \int_{0}^{1} \frac{(1-\tau)^{\alpha-1}}{\Gamma(\alpha)}\left(\int_{0}^{\tau} \frac{(\tau-s)^{\beta-1}}{\Gamma(\beta)} d s\right) d \tau \\
+a\|u-v\| \int_{0}^{1} \frac{(1-\tau)^{\alpha-1}}{\Gamma(\alpha)} \tau^{\alpha-1} d \tau \\
+\left[\int _ { 0 } ^ { \mu } \frac { ( \mu - \tau ) ^ { \alpha - 1 } } { \Gamma ( \alpha ) } \left(\int_{0}^{\tau} \frac{(\tau-s)^{\beta-1}}{\Gamma(\beta)}[L\|u-v\|] d s\right.\right. \\
\left.\left.+a\|u-v\| \int_{0}^{\mu} \frac{(\mu-\tau)^{\alpha-1}}{\Gamma(\alpha)} \tau^{\alpha-1}\right) d \tau\right] \\
=\left(\frac{L\left(1+\mu^{\alpha+\beta}\right)}{\Gamma(\alpha+\beta+1)}+\frac{a \Gamma(\alpha)\left(1+\mu^{2 \alpha-1}\right)}{\Gamma(2 \alpha)}\right)\|u-v\|,
\end{gathered}
$$

it follows that $B$ is a contraction mapping in $B_{r}$. The continuity of $f$ implies that the operator $A$ is continuous. Also, $A$ is uniformly bounded on $B_{r}$ as

$$
\begin{gathered}
=\sup _{t \in[0,1]}\left|\int_{0}^{t} \frac{(t-\tau)^{\alpha-1}}{\Gamma(\alpha)}\left(\int_{0}^{\tau} \frac{(\tau-s)^{\beta-1}}{\Gamma(\beta)}(g(s)-f(s, u(s))) d s-\frac{a}{\tau} u(\tau)\right) d \tau\right| \\
\leq \sup _{t \in[0,1]} \int_{0}^{t} \frac{(t-\tau)^{\alpha-1}}{\Gamma(\alpha)}\left(\int_{0}^{\tau} \frac{(\tau-s)^{\beta-1}}{\Gamma(\beta)}|g(s)+f(s, u(s))| d s+\left|\frac{a}{\tau} u(\tau)\right|\right) d \tau \\
\leq \sup _{t \in[0,1]} \int_{0}^{t} \frac{(t-\tau)^{\alpha-1}}{\Gamma(\alpha)}\left(\int_{0}^{\tau} \frac{(\tau-s)^{\beta-1}}{\Gamma(\beta)} d s\right) d \tau\left[\gamma+\|\sigma\|_{L^{1}}\right] \\
+\sup _{t \in[0,1]} \int_{0}^{t} \frac{(t-\tau)^{\alpha-1}}{\Gamma(\alpha)} \frac{\tau^{\alpha}}{\tau} d \tau a\|u\| \\
\leq\left[\gamma+\|\sigma\|_{\left.L^{1}\right]} \int_{0}^{1} \frac{(1-\tau)^{\alpha-1}}{\Gamma(\alpha)}\left(\int_{0}^{\tau} \frac{(\tau-s)^{\beta-1}}{\Gamma(\beta)} d s\right) d \tau\right. \\
\quad+a\|u\| \int_{0}^{1} \frac{(1-\tau)^{\alpha-1}}{\Gamma(\alpha)} \tau^{\alpha-1} d \tau
\end{gathered}
$$




$$
\leq \frac{\left[\gamma+\|\sigma\|_{L^{1}}\right]}{\Gamma(\alpha+\beta+1)}+\frac{\Gamma(\alpha) a r}{\Gamma(2 \alpha)}
$$

Next we show that $A$ is a compact operator in $B_{r}$. Let $t_{1}, t_{2} \in[0,1]$ we have

$$
\begin{gathered}
\left\|(A u)\left(t_{1}\right)-(A u)\left(t_{2}\right)\right\| \\
=\| \int_{0}^{t_{1}} \frac{\left(t_{1}-\tau\right)^{\alpha-1}}{\Gamma(\alpha)}\left(\int_{0}^{\tau} \frac{(\tau-s)^{\beta-1}}{\Gamma(\beta)}(g(s)-f(s, u(s))) d s+\frac{a}{\tau} u(\tau)\right) d \tau \\
-\int_{0}^{t_{2}} \frac{\left(t_{2}-\tau\right)^{\alpha-1}}{\Gamma(\alpha)}\left(\int_{0}^{\tau} \frac{(\tau-s)^{\beta-1}}{\Gamma(\beta)}(g(s)-f(s, u(s))) d s+\frac{a}{\tau} u(\tau)\right) d \tau \| \\
\leq \frac{(\gamma+\|f\|)}{\Gamma(\alpha+\beta+1)}\left|t_{1}^{\alpha+\beta}-t_{2}^{\alpha+\beta}\right|+\frac{a \Gamma(\alpha)}{\Gamma(2 \alpha)}\left|t_{1}^{2 \alpha-1}-t_{2}^{2 \alpha-1}\right|
\end{gathered}
$$

which is independent of $u$. Thus, $A$ is equicontinuous. We used the fact that $f$ maps bounded subsets into relatively compact subsets. Therefore, $A$ is relatively compact on $B_{r}$. Hence, by the Arzela- Ascoli theorem, $A$ is compact on $B_{r}$. Thus all the assumptions of Theorem 1 are satisfied and the conclusion of Theorem 1 implies that the boundary value problem 2.1 has at least one solution on $[0,1]$. This completes the proof.

\section{ApPLiCATIONS}

In this section, we illustrate some applications of the theory established in this paper.

Example 1. Consider the following (BVP)

It is clear that

$$
\begin{gathered}
D^{1 / 2}\left(D^{1 / 2}+\frac{0.1}{t}\right) u(t)+\frac{1}{(t+2)^{2}} \frac{|u|}{1+|u|}=t, \quad t \in(0,1) \\
u(0)=u\left(\frac{1}{2}\right)=u(1)=0 .
\end{gathered}
$$

and $\sup _{t \in(0,1)} g(t)=1$. Moreover, we have

$\ell=\frac{L}{\Gamma(\alpha+\beta+1)}\left(2+\mu^{\alpha+\beta}\right)+a \frac{\Gamma(\alpha)}{\Gamma(2 \alpha)}\left(2+\mu^{2 \alpha-1}\right)=0.625+0.3544=0.9794<1$

Thus, by Theorem 2 , the boundary value problem 4.1 has a unique solution on $[0,1]$.

Example 2. Consider the following (BVP)

$$
\begin{gathered}
D^{1 / 4}\left(D^{1 / 2}+\frac{0.1}{t}\right) u(t)+\frac{1}{(t+2)^{2}} \frac{|u|}{1+|u|}=t, \quad t \in(0,1) \\
u(0)=u\left(\frac{3}{4}\right)=u(1)=0 .
\end{gathered}
$$


It is clear that $|f(t, u)| \leq \frac{1}{(t+2)^{2}}:=\sigma(t)$,

$$
|f(t, u)-f(t, v)| \leq \frac{1}{4}|u-v|,
$$

and $\sup _{t \in(0,1)} g(t)=1$. Moreover, we have

$$
\begin{gathered}
\left(\frac{L\left(1+\mu^{\alpha+\beta}\right)}{\Gamma(\alpha+\beta+1)}+\frac{a \Gamma(\alpha)\left(1+\mu^{2 \alpha-1}\right)}{\Gamma(2 \alpha)}\right) \\
=\frac{1.8}{3 \Gamma\left(\frac{3}{4}\right)}+0.1772=0.5+0.1772=0.6772<1 .
\end{gathered}
$$

Thus, by Theorem 3, the boundary value problem 4.2 has at least one solution on [0, 1]. If $\mu=\frac{1}{3}$, we obtain

$$
\left(\frac{L\left(1+\mu^{\alpha+\beta}\right)}{\Gamma(\alpha+\beta+1)}+\frac{a \Gamma(\alpha)\left(1+\mu^{2 \alpha-1}\right)}{\Gamma(2 \alpha)}\right)=1.07>1 ;
$$

hence the problem has not a solution.

Note that when $a \geq 1$ the (BVPS) 4.1 and 4.2 have no solutions in [0,1] :

Example 3. Consider the following (BVP)

$$
\begin{gathered}
D^{\beta}\left(D^{\alpha}+\frac{1}{t}\right) u(t)+\frac{1}{(t+2)^{2}} \frac{|u|}{1+|u|}=t, \quad t \in(0,1) \\
u(0)=u(\mu)=u(1)=0 .
\end{gathered}
$$

It is clear that $|f(t, u)| \leq \frac{1}{(t+2)^{2}}=\sigma(t)$,

$$
|f(t, u)-f(t, v)| \leq \frac{1}{4}|u-v|,
$$

and $\sup _{t \in(0,1)} g(t)=1$. Moreover, since $\Gamma(\alpha)>\Gamma(2 \alpha)$ for $0<\alpha \leq 1$, we have

$$
\left(\frac{L\left(1+\mu^{\alpha+\beta}\right)}{\Gamma(\alpha+\beta+1)}+\frac{a \Gamma(\alpha)\left(1+\mu^{2 \alpha-1}\right)}{\Gamma(2 \alpha)}\right)>1, \quad \forall 0<\alpha, \beta \leq 1 .
$$

We have the following proposition:

Proposition 1. If $a \geq 1$, then the solution of the boundary value problem 2.1 does not exist for all $0<\alpha, \beta \leq 1$. 


\section{CONCLUSiOnS}

The existence of solutions for a Dirichlet boundary value problem involving LaneEmden equation with two different fractional orders has been discussed. We apply the concepts of fractional calculus together with fixed point theorems to establish the existence results. First of all, we find the unique solution for a linear Dirichlet boundary value problem involving Lane-Emden equation with two different fractional orders, which in fact provides the platform to prove the existence of solutions for the associated nonlinear fractional Lane-Emden equation with two different orders. Our approach is applicable to a variety of real world problems.

\section{REFERENCES}

[1] H. Adibi and A. M. Rismani, "On using a modified Legendre-spectral method for solving singular IVPs of Lane-Emden type," Comput. Math. Appl., vol. 60, no. 7, pp. 2126-2130, 2010.

[2] R. P. Agarwal and D. O'Regan, "Singular boundary value problems for superlinear second order ordinary and delay differential equations," J. Differ. Equations, vol. 130, no. 2, pp. 333-355, 1996.

[3] R. P. Agarwal and D. O'Regan, "Existence theory for single and multiple solutions to singular positone boundary value problems," J. Differ. Equations, vol. 175, no. 2, pp. 393-414, 2001.

[4] R. P. Agarwal and D. O'Regan, "Existence theory for singular initial and boundary value problems: A fixed point approach,” Appl. Anal., vol. 81, no. 2, pp. 391-434, 2002.

[5] R. P. Agarwal, D. O’Regan, and V. Lakshmikantham, "Quadratic forms and nonlinear nonresonant singular second order boundary value problems of limit circle type," Z. Anal. Anwend., vol. 20, no. 3, pp. 727-737, 2001.

[6] A. Bhrawy and A. Alofi, "A Jacobi-Gauss collocation method for solving nonlinear Lane-Emden type equations,” Commun. Nonlinear Sci. Numer. Simulat., vol. 17, no. 1, pp. 62-70, 2012.

[7] B. Bonilla, M. Rivero, and J. J. Trujillo, "On systems of linear fractional differential equations with constant coefficients," Appl. Math. Comput., vol. 187, no. 1, pp. 68-78, 2007.

[8] S. Chandrasekhar, An introduction to the study of stellar structure. New York: Dover Publications, Inc., 1957.

[9] M. S. H. Chowdhury and I. Hashim, "Solutions of Emden-Fowler equations by homotopyperturbation method," Nonlinear Anal., Real World Appl., vol. 10, no. 1, pp. 104-115, 2009.

[10] M. M. Coclite and G. Palmieri, "On a singular nonlinear Dirichlet problem," Commun. Partial Differ. Equations, vol. 14, no. 10, pp. 1315-1327, 1989.

[11] R. Emden, Gaskugeln. Anwendung der mechanischen Wärmetheorie auf kosmologische und meteorologische Probleme. Leipzig: B. G. Teubner, 1907.

[12] R. W. Ibrahim, "Global controllability of a set of fractional differential equations," Math. Notes, Miskolc, vol. 12, no. 1, pp. 51-60, 2011.

[13] R. W. Ibrahim and M. Darus, "Subordination and superordination for analytic functions involving fractional integral operator," Complex Var. Elliptic Equ., vol. 53, no. 11, pp. 1021-1031, 2008.

[14] R. W. Ibrahim and M. Darus, "Subordination and superordination for univalent solutions for fractional differential equations," J. Math. Anal. Appl., vol. 345, no. 2, pp. 871-879, 2008.

[15] R. W. Ibrahim and H. A. Jalab, "Existence of the solution of fractional integral inclusion with time delay," Math. Notes, Miskolc, vol. 11, no. 2, pp. 139-150, 2010.

[16] R. W. Ibrahim and S. Momani, "On the existence and uniqueness of solutions of a class of fractional differential equations," J. Math. Anal. Appl., vol. 334, no. 1, pp. 1-10, 2007.

[17] A. A. Kilbas, H. M. Srivastava, and J. J. Trujillo, Theory and applications of fractional differential equations. Amsterdam: North-Holland Mathematics Studies, Elsevier, 2006, vol. 204. 
[18] J. H. Lane, "On the theoretical temperature of the sun under the hypothesis of a gaseous mass maintaining its volume by its internal heat and depending on the laws of gases known to terrestrial experiment," The American Journal of Science and Arts, vol. 50, no. 2, pp. 57-74, 1870.

[19] R. Lewandowski and B. Chorazyczewski, "Identification of the parameters of the Kelvin-Voigt and the Maxwell fractional models, used to modeling of viscoelastic dampers," Computers and Structures, vol. 88, pp. 1-17, 2010.

[20] S. Momani and R. W. Ibrahim, "On a fractional integral equation of periodic functions involving Weyl-Riesz operator in Banach algebras," J. Math. Anal. Appl., vol. 339, no. 2, pp. 1210-1219, 2208.

[21] E. Momoniat and C. Harley, "An implicit series solution for a boundary value problem modelling a thermal explosion," Math. Comput. Modelling, vol. 53, no. 1-2, pp. 249-260, 2011.

[22] K. Parand, M. Dehghan, A. R. Rezaei, and S. M. Ghaderi, "An approximation algorithm for the solution of the nonlinear Lane-Emden type equations arising in astrophysics using Hermite functions collocation method," Comput. Phys. Commun., vol. 181, no. 6, pp. 1096-1108, 2010.

[23] K. Parand and A. Pirkhedri, "Sinc-collocation method for solving astrophysics equations," New Astron, vol. 15, pp. 533-537, 2010.

[24] I. Podlubny, Fractional differential equations. An introduction to fractional derivatives, fractional differential equations, to methods of their solution and some of their applications. San Diego, CA: Academic Press, 1999.

[25] D. R. Smart, Fixed point theorems, ser. Cambridge Tracts in Mathematics. Cambridge: Cambridge University Press, 1980, vol. 66.

[26] A. Yıldırım and T. Öziş, "Solutions of singular IVPs of Lane-Emden type by the variational iteration method," Nonlinear Anal., Theory Methods Appl., vol. 70, no. 6, pp. 2480-2484, 2009.

[27] F. Yu, "Integrable coupling system of fractional soliton equation hierarchy," Phys. Lett., A, vol. 373, no. 41, pp. 3730-3733, 2009.

\section{Author's address}

\section{Rabha W. Ibrahim}

Institute of Mathematical Sciences, University Malaya, 50603, Kuala Lumpur, Malaysia

E-mail address: rabhaibrahimeyahoo.com 